\title{
Individual and cumulative effect of type 2 diabetes genetic susceptibility variants on risk of coronary heart disease
}

\author{
R. Pfister • D. Barnes • R. N. Luben • K.-T. Khaw • \\ N. J. Wareham • C. Langenberg
}

Received: 26 January 2011 / Accepted: 12 May 2011 /Published online: 3 June 2011

(C) Springer-Verlag 2011

\begin{abstract}
Aims/hypothesis Type 2 diabetes is a major risk factor for CHD. We hypothesised that diabetes genetic susceptibility variants might be associated with increased CHD risk.

Methods We examined the individual and cumulative effect of 38 common genetic variants previously reported to be associated with type 2 diabetes on risk of incident CHD in 20,467 participants of the European Prospective Investigation into Cancer and Nutrition (EPIC) Norfolk Study who had been free of CHD at baseline.

Results During a mean follow-up of 10.7 years, 2,190 participants had a CHD event. Two individual variants next to the TSPAN8 (HR 1.07, 95\% CI 1.00-1.14) and the $C D K N 2 A / B$ region $(1.11,1.04-1.17)$ were significantly associated with increased CHD risk. A genetic score based on the 38 diabetes variants was significantly associated with an increased risk of CHD (1.08, 1.01-1.14 per score tertile). Adjustment for prevalent and incident diabetes
\end{abstract}

Electronic supplementary material The online version of this article (doi:10.1007/s00125-011-2206-5) contains unedited supplementary material, which is available to authorised users.

R. Pfister $\cdot$ D. Barnes $\cdot$ N. J. Wareham $\cdot$ C. Langenberg $(\bowtie)$

MRC Epidemiology Unit, Institute of Metabolic Science,

Addenbrooke's Hospital,

Box 285, Hills Road,

Cambridge CB2 0QQ, UK

e-mail: Claudia.Langenberg@mrc-epid.cam.ac.uk

R. Pfister

Department III of Internal Medicine, Herzzentrum,

University of Cologne,

Cologne, Germany

R. N. Luben $\cdot$ K.-T. Khaw

Department of Public Health and Primary Care, Institute of Public Health, University of Cambridge,

Cambridge, UK attenuated the association of the TSPAN8 variant (1.06, $0.99-1.13)$ and the genetic score $(1.05,0.99-1.12$ per score tertile) with CHD risk, but not that of the $C D K N 2 A / B$ variant (1.11, 1.05-1.18). Addition of the genetic score did not improve risk discrimination based on clinical risk factors.

Conclusions/interpretation The increased risk of CHD observed with genetic susceptibility to type 2 diabetes was at least partly mediated by its diabetes-predisposing effect and was not useful for clinical risk discrimination. The potential role of pathways associated with the variant $C D K N 2 A / B$ in linking diabetes and CHD needs further exploration.

Keywords Coronary heart disease - Genetic score - Single nucleotide polymorphism Type 2 diabetes
Abbreviations
EPIC European Prospective Investigation into Cancer and Nutrition
GWAS Genome-wide association studies
LD Linkage disequilibrium

\section{Introduction}

A steadily increasing number of common genetic variants associated with type 2 diabetes mellitus has been reported (electronic supplementary material [ESM] Table 1). Several of these variants are within genes that are plausible candidates for regulation of gluco-metabolic processes, and thus provide insight into the pathogenesis of diabetes.

Diabetes is a major risk factor for CHD, but the underlying pathophysiological mechanisms are largely 
unclear. Due to the strong and presumably causal association between diabetes and CHD, variants that are associated with risk of diabetes would be expected to also be associated with increased risk of CHD. If confirmed, this would have several implications. Diabetes variants that are more strongly related to CHD risk might point to associated pathways that mechanistically link diabetes to CHD. So far few type 2 diabetes variants have been examined for their association with cardiovascular outcome and the results are inconsistent [1-3]. Furthermore, although the effect of individual genetic variants on diabetes risk is small, an aggregate of many genetic variants combined to a score might substantially influence risk of diabetes and in consequence risk of CHD. The independent contribution of genetic diabetes susceptibility to CHD risk has not been examined.

We therefore examined the association between individual type 2 diabetes variants and risk of incident CHD events and the risk associated with a genetic predisposition score in participants of the European Prospective Investigation into Cancer and Nutrition (EPIC) Norfolk Study [4].

\section{Methods}

EPIC-Norfolk is a prospective population-based study of 25,663 men and women aged 40 to 79 years and living in Norfolk, UK; it has been described in detail elsewhere [4]. Baseline samples of DNA were available for genotyping in 21,323 participants. We excluded 165 participants with selfreport of non-European descent and 691 participants with baseline history of myocardial infarction. Incident cases of diabetes were ascertained as previously described [5] using multiple sources of evidence, including self-report, and linkage to primary care registers, secondary care registers, hospital admissions and mortality data. Incident cases of CHD and cardiovascular disease were defined by the corresponding 'International Classification of Disease' codes on death certificates and hospital discharge data, which have proven to be accurate in an earlier validation study [6]. We report results with follow-up to March 2007. The study was approved by the Norwich District Health Authority Ethics Committee and all participants gave signed, informed consent.

We genotyped 42 autosomal type 2 diabetes variants reported in European-origin populations at a genome-wide association significance level of $5 \times 10^{-8}$, or with consistent replication in several cohorts or proxies in high linkage disequilibrium (LD) (ESM Table 1). Genotypes were obtained from the iPLEX (Sequenom, San Diego, CA, USA) platform or Custom TaqMan SNP Genotyping Assays (Applied Biosystems, Foster City, CA, USA). Four variants did not pass genotyping quality control criteria and were excluded from analyses.
The diabetes-risk-increasing alleles were determined on the basis of recent literature (ESM Table 1). A genetic predisposition score was calculated for each participant by adding the diabetes risk-increasing alleles from all 38 variants. We also calculated a weighted score based on the effect size (OR) obtained from previous replication analyses (ESM Table 1). Logistic regression was used to calculate the risk (OR) of individual variants and the genetic score for combined prevalent and incident diabetes. Cox proportional hazard regression was used to examine the risk (HR) of incident CHD. All reported $p$ values were not corrected for multiple testing unless indicated otherwise. Harrell's $c$-statistics were calculated to estimate the risk discrimination of models with and without the genetic score. In sensitivity analyses, we calculated a genetic score by: (1) excluding the four variants with an OR for diabetes of less than 1 in our cohort; and (2) standardising the score in persons with $<50 \%$ missing genotypes to those for persons with complete data. We observed similar results for both scores as in primary analysis (data not shown).

All analyses were performed using STATA version 10.1 (Statacorp, College Station, TX, USA). We had 96\%/82\% power to detect a relative risk of $1.2 / 1.15$ of individual variants for incident CHD with an $\alpha$ level at $p=0.05$.

\section{Results}

During a mean follow-up of 10.7 years, 2,190 (10.7\%) of 20,467 participants had an incident CHD event. Baseline characteristics are shown in Table 1.

Table 2 shows the associations of 38 type 2 diabetes variants with risk of prevalent and incident diabetes and

Table 1 Baseline characteristics according to incident CHD

\begin{tabular}{llll}
\hline Characteristic & $\begin{array}{l}\text { With } \\
\text { incident } \\
\text { CHD }\end{array}$ & $\begin{array}{l}\text { Without } \\
\text { incident } \\
\text { CHD }\end{array}$ & Total \\
\hline$n$ & 2,190 & 18,277 & 20,467 \\
Age (years) & $64.5 \pm 8.0$ & $58.0 \pm 9.2$ & $58.7 \pm 9.3$ \\
Male sex (\%) & 65.7 & 46.1 & 48.2 \\
BMI (kg/m $\left.{ }^{2}\right)$ & $27.2 \pm 3.8$ & $26.2 \pm 3.8$ & $26.3 \pm 3.8$ \\
Systolic BP (mmHg) & $144 \pm 19$ & $135 \pm 18$ & $136 \pm 18$ \\
Cholesterol (mmol/1) & $6.45 \pm 1.20$ & $6.14 \pm 1.16$ & $6.17 \pm 1.17$ \\
Smoking (\%) & 66.5 & 52.4 & 53.9 \\
Family history & & & \\
Myocardial infarction (\%) & 43.5 & 35.3 & 36.2 \\
Diabetes (\%) & 11.7 & 13.1 & 12.9 \\
Prevalent diabetes (\%) & 8.1 & 2.5 & 3.1 \\
Incident diabetes (\%) & 6.8 & 2.7 & 3.1 \\
\hline
\end{tabular}

Data presented as mean $\pm \mathrm{SD}$ or percentage 
Table 2 Age- and sex-adjusted association of diabetes susceptibility variants with risk of type 2 diabetes mellitus and incident CHD in EPICNorfolk 1993-2007 (in ascending order of previously reported effect size on type 2 diabetes)

\begin{tabular}{|c|c|c|c|c|c|c|c|c|c|}
\hline Locus & $\mathrm{SNP}^{\mathrm{a}}$ & Effect allele $\mathrm{e}^{\mathrm{b}} / \mathrm{other}$ & $\operatorname{RAF}^{\mathrm{c}}(\%)$ & $\begin{array}{l}\text { OR for T2 } D^{d} \\
(95 \% \mathrm{CI})\end{array}$ & $p$ value & $\begin{array}{l}\text { HR for } \mathrm{CHD}^{\mathrm{e}} \\
(95 \% \mathrm{CI})\end{array}$ & $p$ value & $\begin{array}{l}\mathrm{HR} \text { for } \mathrm{CHD}^{\mathrm{e}, \mathrm{f}} \\
(95 \% \mathrm{CI})\end{array}$ & $p$ value \\
\hline$V E G F A$ & rs 9472138 & $\mathrm{~T} / \mathrm{C}$ & 29.8 & $1.05(0.96-1.14)$ & 0.33 & $1.02(0.96-1.09)$ & 0.53 & $1.02(0.95-1.09)$ & 0.60 \\
\hline$O A S L / H N F 1 A$ & rs7957197 & $\mathrm{T} / \mathrm{A}$ & 80.8 & $1.00(0.90-1.11)$ & 0.97 & $1.01(0.94-1.09)$ & 0.77 & $1.01(0.94-1.09)$ & 0.73 \\
\hline ZFAND6 & rs 11634397 & $\mathrm{G} / \mathrm{A}$ & 66.6 & $0.99(0.91-1.08)$ & 0.86 & $0.99(0.93-1.05)$ & 0.73 & $0.99(0.93-1.05)$ & 0.73 \\
\hline TP53INP1 & rs896854 & $\mathrm{T} / \mathrm{C}$ & 49.7 & $1.08(1.00-1.17)$ & 0.05 & $0.96(0.90-1.02)$ & 0.17 & $0.96(0.90-1.01)$ & 0.13 \\
\hline ADAMTS9 & rs 4607103 & $\mathrm{C} / \mathrm{T}$ & 76.3 & $0.98(0.89-1.08)$ & 0.68 & $1.00(0.94-1.07)$ & 0.94 & $1.01(0.94-1.08)$ & 0.88 \\
\hline$D C D$ & rs 1153188 & $\mathrm{~A} / \mathrm{T}$ & 73.4 & $1.07(0.97-1.17)$ & 0.18 & $1.02(0.95-1.09)$ & 0.62 & $1.01(0.95-1.08)$ & 0.72 \\
\hline$P R C 1$ & rs 8042680 & $\mathrm{~A} / \mathrm{C}$ & 31.3 & $1.04(0.96-1.14)$ & 0.32 & $1.05(0.98-1.12)$ & 0.15 & $1.05(0.98-1.12)$ & 0.15 \\
\hline$S H B G$ & rs1799941 & $\mathrm{G} / \mathrm{A}$ & 74.1 & $1.10(1.00-1.20)$ & 0.06 & $1.01(0.95-1.09)$ & 0.68 & $1.01(0.94-1.08)$ & 0.85 \\
\hline$D G K B / T M E M 195^{\mathrm{g}}$ & rs2191349 & $\mathrm{T} / \mathrm{G}$ & 54.1 & $1.06(0.97-1.15)$ & 0.18 & $0.98(0.92-1.04)$ & 0.53 & $0.98(0.92-1.04)$ & 0.49 \\
\hline GCKR & rs780094 & $\mathrm{C} / \mathrm{T}$ & 60.8 & $1.09(1.01-1.19)$ & 0.04 & $1.05(0.99-1.12)$ & 0.10 & $1.05(0.99-1.12)$ & 0.12 \\
\hline$K C N Q 1$ & rs231362 & $\mathrm{G} / \mathrm{A}$ & 51.9 & $1.08(1.00-1.17)$ & 0.06 & $0.95(0.90-1.01)$ & 0.10 & $0.95(0.89-1.01)$ & 0.07 \\
\hline$G C K$ & rs4607517 & $\mathrm{A} / \mathrm{G}$ & 17.8 & $1.09(0.98-1.20)$ & 0.11 & $1.00(0.93-1.08)$ & 0.94 & $1.00(0.92-1.08)$ & 0.98 \\
\hline$H M G A 2$ & rs 1531343 & $\mathrm{C} / \mathrm{G}$ & 10.3 & $0.99(0.87-1.13)$ & 0.87 & $1.01(0.92-1.11)$ & 0.86 & $1.02(0.92-1.12)$ & 0.76 \\
\hline$T L E 4 / C H C H D 9^{\mathrm{h}}$ & rs13292136 & $\mathrm{C} / \mathrm{T}$ & 93.1 & $1.04(0.88-1.22)$ & 0.65 & $1.02(0.91-1.15)$ & 0.74 & $1.01(0.90-1.14)$ & 0.81 \\
\hline$B C L 11 A$ & rs243021 & $\mathrm{A} / \mathrm{G}$ & 46.5 & $1.12(1.03-1.22)$ & 0.01 & $0.98(0.93-1.05)$ & 0.62 & $0.98(0.92-1.04)$ & 0.42 \\
\hline ADAM30 & rs2641348 & $\mathrm{G} / \mathrm{A}$ & 10.5 & $1.07(0.94-1.21)$ & 0.32 & $1.02(0.93-1.13)$ & 0.65 & $1.02(0.92-1.12)$ & 0.74 \\
\hline TSPAN8 & rs7961581 & $\mathrm{C} / \mathrm{T}$ & 28.3 & $1.06(0.97-1.16)$ & 0.18 & $1.07(1.00-1.14)$ & 0.04 & $1.06(0.99-1.13)$ & 0.08 \\
\hline$J A Z F 1$ & rs 864745 & $\mathrm{~T} / \mathrm{C}$ & 49.4 & $1.09(1.00-1.18)$ & 0.04 & $1.03(0.97-1.09)$ & 0.37 & $1.02(0.96-1.08)$ & 0.56 \\
\hline IRSI & rs 7578326 & $\mathrm{~A} / \mathrm{G}$ & 65.5 & $1.12(1.02-1.22)$ & 0.01 & $1.06(1.00-1.13)$ & 0.06 & $1.06(0.99-1.13)$ & 0.08 \\
\hline$S Y N 2 / P P A R G$ & rs17036101 & $\mathrm{G} / \mathrm{A}$ & 93.9 & $1.08(0.90-1.28)$ & 0.42 & $0.96(0.85-1.09)$ & 0.52 & $0.95(0.84-1.08)$ & 0.46 \\
\hline$M T N R I B$ & rs 10830963 & $\mathrm{G} / \mathrm{C}$ & 27.7 & $1.05(0.96-1.15)$ & 0.26 & $0.97(0.90-1.04)$ & 0.35 & $0.96(0.90-1.03)$ & 0.22 \\
\hline NOTCH2 & rs10923931 & $\mathrm{T} / \mathrm{G}$ & 10.3 & $1.06(0.93-1.21)$ & 0.38 & $1.01(0.92-1.11)$ & 0.85 & $1.01(0.91-1.11)$ & 0.90 \\
\hline WFS1 & rs10010131 & $\mathrm{G} / \mathrm{A}$ & 59.2 & $1.10(1.01-1.19)$ & 0.03 & $1.02(0.96-1.08)$ & 0.53 & $1.01(0.95-1.07)$ & 0.77 \\
\hline SLC $30 A 8$ & rs 13266634 & $\mathrm{C} / \mathrm{T}$ & 69.4 & $1.05(0.96-1.15)$ & 0.27 & $1.02(0.95-1.09)$ & 0.60 & $1.01(0.94-1.08)$ & 0.83 \\
\hline$C D K N 2 A / B$ & rs564398 & $\mathrm{T} / \mathrm{C}$ & 57.6 & $0.98(0.91-1.07)$ & 0.68 & $1.11(1.04-1.17)$ & 0.001 & $1.11(1.05-1.18)$ & 0.001 \\
\hline THADA & rs7578597 & $\mathrm{T} / \mathrm{C}$ & 88.6 & $1.20(1.05-1.37)$ & 0.01 & $0.96(0.87-1.05)$ & 0.34 & $0.94(0.86-1.03)$ & 0.19 \\
\hline$A D C Y 5$ & rs 11708067 & $\mathrm{~A} / \mathrm{G}$ & 76.3 & $1.11(1.01-1.23)$ & 0.03 & $1.06(0.99-1.14)$ & 0.09 & $1.06(0.99-1.13)$ & 0.12 \\
\hline API5 & rs9300039 & $\mathrm{C} / \mathrm{A}$ & 89.4 & $1.04(0.91-1.18)$ & 0.59 & $0.94(0.86-1.04)$ & 0.22 & $0.94(0.85-1.03)$ & 0.18 \\
\hline HHEX & rs 1111875 & $\mathrm{C} / \mathrm{T}$ & 59.0 & $1.07(0.99-1.17)$ & 0.09 & $1.00(0.95-1.07)$ & 0.90 & $0.99(0.93-1.05)$ & 0.80 \\
\hline KCNJ11 & rs5219 & $\mathrm{T} / \mathrm{C}$ & 36.3 & $1.05(0.96-1.14)$ & 0.26 & $1.02(0.96-1.08)$ & 0.60 & $1.01(0.95-1.08)$ & 0.68 \\
\hline PPARG & rs1801282 & $\mathrm{C} / \mathrm{G}$ & 88.0 & $1.15(1.01-1.32)$ & 0.03 & $0.93(0.85-1.02)$ & 0.11 & $0.92(0.84-1.01)$ & 0.07 \\
\hline CENTD $2^{\mathrm{i}}$ & rs 1552224 & $\mathrm{~A} / \mathrm{C}$ & 84.0 & $1.21(1.08-1.36)$ & 0.002 & $1.00(0.92-1.08)$ & 0.93 & $0.99(0.91-1.07)$ & 0.79 \\
\hline$N P P B$ & rs198389 & $\mathrm{A} / \mathrm{G}$ & 57.5 & $1.02(0.94-1.11)$ & 0.64 & $1.02(0.96-1.09)$ & 0.44 & $1.02(0.96-1.09)$ & 0.46 \\
\hline$C D K N 2 A / B$ & rs10811661 & $\mathrm{T} / \mathrm{C}$ & 82.1 & $1.12(1.00-1.25)$ & 0.04 & $1.05(0.97-1.13)$ & 0.26 & $1.04(0.96-1.13)$ & 0.33 \\
\hline$I G F 2 B P 2$ & rs 4402960 & $\mathrm{~T} / \mathrm{G}$ & 31.4 & $1.11(1.02-1.21)$ & 0.02 & $0.99(0.92-1.05)$ & 0.67 & $0.97(0.91-1.04)$ & 0.38 \\
\hline$C D K A L 1$ & rs 7756992 & $\mathrm{G} / \mathrm{A}$ & 26.5 & $1.17(1.06-1.21)$ & $9.9 \times 10^{-4}$ & $1.06(0.99-1.13)$ & 0.11 & $1.05(0.98-1.12)$ & 0.19 \\
\hline$K C N Q 1$ & rs 2237892 & $\mathrm{C} / \mathrm{T}$ & 94.2 & $1.10(0.92-1.31)$ & 0.32 & $1.03(0.91-1.18)$ & 0.61 & $1.03(0.90-1.17)$ & 0.67 \\
\hline$T C F 7 L 2$ & rs7903146 & $\mathrm{T} / \mathrm{C}$ & 29.2 & $1.64(1.34-1.59)$ & $9.5 \times 10^{-18}$ & $1.05(0.98-1.12)$ & 0.17 & $1.02(0.95-1.09)$ & 0.58 \\
\hline \multicolumn{10}{|l|}{ Gene score } \\
\hline Tertile & & & & $1.42(1.30-1.55)$ & $1.9 \times 10^{-15}$ & $1.08(1.01-1.14)$ & 0.02 & $1.05(0.99-1.12)$ & 0.09 \\
\hline Tertile, weighted & & & & $1.50(1.38-1.64)$ & $2.5 \times 10^{-20}$ & $1.07(1.01-1.13)$ & 0.03 & $1.04(0.98-1.10)$ & 0.19 \\
\hline
\end{tabular}

${ }^{\text {a }}$ SNP, single nucleotide polymorphism

${ }^{\mathrm{b}}$ According to type 2 diabetes risk-increasing allele in original reports

${ }^{\mathrm{c}} \mathrm{RAF}$, risk allele frequency in participants without known type 2 diabetes

${ }^{\mathrm{d}}$ OR for type 2 diabetes (T2D) is for combined prevalent $(n=640)$ and incident $(n=640)$ cases per allele or gene score tertile

${ }^{\mathrm{e}} \mathrm{HR}$ is per type 2 diabetes risk allele or gene score tertile, minimum events $n=2,043$, total participants $n=19,201$ for individual variants, $n=1,573$ and $n=14,871$ respectively for the gene score

${ }^{\mathrm{f}}$ Adjusted for prevalent and incident type 2 diabetes

${ }^{\mathrm{g}}$ TMEM195 (also known as AGMO); ${ }^{\mathrm{h}}$ CHCHD9 (also known as CHCHD2P9); ${ }^{\mathrm{i}}$ CENTD2 (also known as ARAP1) 
incident CHD. In our population individual diabetes variants showed an effect direction for risk of diabetes that was consistent with the original reports in 34 of the 38 variants. The risk of incident CHD ranged from HR 0.93 to 1.11 per diabetes risk allele across variants. Two variants, one next to the $C D K N 2 A / B$ locus and one next to the TSPAN8 locus, showed a significant association with CHD, with the same direction of effect as reported for diabetes. When correcting for multiple testing, the association of the $C D K N 2 A / B$ variant with $C H D$ remained significant $(p=0.04)$ but not that of the TSPAN8 variant.

The genetic score was significantly associated with risk of CHD, with each diabetes risk allele associated with a $1.5 \%$ $(95 \%$ CI $0.2-2.8, p=0.03)$ risk increase for CHD in age- and sex-adjusted analysis. However, there was no significant association between the genetic score and risk of cardiovascular disease (HR 1.005, 95\% CI 0.997-1.013, $p=0.22$ ).

Adjusting for prevalent and incident diabetes did not attenuate the association of the $C D K N 2 A / B$ variant (HR 1.11, 95\% CI 1.05-1.18, $p=0.001$ ), but did attenuate that of the TSPAN8 variant (HR 1.06, 95\% CI 0.99-1.13, $p=0.08$ ) and the genetic score (HR 1.05, 95\% CI 0.99-1.12, $p=0.09$ ) with CHD risk (Table 2). Additional adjustment for BMI did not change results (data not shown).

The $c$-statistic for predicting a CHD event was unchanged by addition of the genetic score to a model including age and sex $(0.73)$ or to a model including age, sex and traditional risk factors $(0.76)$.

\section{Discussion}

We demonstrated a significant association of the $C D K N 2 A / B$ and TSPAN8 variants, and a genetic score built with 38 diabetes variants with risk of CHD. The association was mediated by the predisposition to diabetes for the TSPAN8 variant and the genetic score. Addition of the genetic score did not improve clinical prediction of CHD.

A recent analysis of the Women's Health Study failed to show an improvement in cardiovascular risk prediction when adding a genetic score of variants associated with cardiovascular disease or intermediate traits [2]. However, the number of events was low $(n=777)$ and the results are difficult to interpret, as different cardiovascular endpoints and genetic variants with contrary effects on distinct risk factors were lumped together. We observed a much stronger association of our diabetes-specific risk score with CHD than with cardiovascular disease. However, although the association between genetic diabetes susceptibility and CHD can be demonstrated, the magnitude of that association is too low to be of clinical use in the prediction of CHD.

The individual variants we identified to be associated with CHD were not those with the strongest association with diabetes, suggesting that these variants or associated pathways might be of particular relevance to the development of CHD. However, the observed association of the TSPAN8 variant with CHD was partly mediated by its association with diabetes and attenuated to borderline significance in the diabetes-adjusted model. Replication of our finding in a larger sample size may help to establish whether or not the TSPAN8 locus is associated with CHD independently of diabetes.

The $C D K N 2 A / B$ variant on chromosome $9 \mathrm{p} 21$ has been previously reported to be associated with CHD risk [7]. The 9 p21 region is under intense research, since several variants within this region have been independently reported in genome-wide association studies (GWAS) for type 2 diabetes and CHD. Several of these variants, including our $C D K N 2 A / B$ variant, were shown to disrupt transcription factor bindingsites and indeed one of the CHD variants had functional relevance for a pathway associated with atherosclerosis in human endothelial cells [8]. Our $C D K N 2 A / B$ variant is in weak LD with one of the CHD variants $\left(r^{2}=0.24\right.$ for rs10116277), which might explain the association with CHD risk. In support of this, we did not observe attenuation after adjustment for diabetes in our study. In contrast, in diabetic patients a significant interaction was found between a variant within the $9 \mathrm{p} 21$ region, which is in LD with our $C D K N 2 A / B$ variant $\left(r^{2}=0.16\right)$, and poor glycaemic control leading to an effect on risk of CHD [9]. This suggests not only separate effects on diabetes and CHD, but a mechanistic link between both diseases within this region.

The IRS1 variant showed a borderline significant association with CHD here and is in high LD $\left(r^{2}=0.93\right)$ with a variant reported in a GWAS of CHD [10]. Notably, the association between the IRS1 variant and CHD here was not attenuated by adjustment for diabetes, suggesting that a potential common pathway for development of diabetes and CHD is associated with this variant.

In conclusion, the increased risk of CHD associated with aggregate genetic diabetes susceptibility was partly mediated by predisposition to diabetes and did not improve clinical risk prediction. The identification of individual variants associated with CHD risk independently of their diabetes predisposition might help to provide insight into the pathophysiological link between diabetes and CHD.

Acknowledgements We thank all of the participants in this study and the EPIC-Norfolk study staff at the University of Cambridge, Department of Public Health and Primary Care. This study was supported by grants (to R. Pfister) from Koeln Fortune Program and Marga- und Walter-BollStiftung, and by grants (to the EPIC-Norfolk Study) from the Medical Research Council UK and Cancer Research UK.

R.P. contributed analyses, interpretation of results and manuscript draft, D.B. contributed statistical analyses and revision of manuscript draft, R.N.L. contributed data collection and management, analyses and revision of manuscript draft, K.-T.K. contributed study design, 
results interpretation and revision of manuscript draft, N.J.W. contributed data acquisition, study design and revision of manuscript draft, C.L. contributed study design and manuscript draft. R.P., D.B., R.N.L., K.-T.K., N.J.W. and C.L. approved the final version of the manuscript to be published.

Duality of interest The authors declare that there is no duality of interest associated with this manuscript.

\section{References}

1. Bielinski SJ, Pankow JS, Folsom AR, North KE, Boerwinkle E (2008) TCF7L2 single nucleotide polymorphisms, cardiovascular disease and all-cause mortality: the Atherosclerosis Risk in Communities (ARIC) Study. Diabetologia 51:968-970

2. Paynter NP, Chasman DI, Pare G et al (2010) Association between a literature-based genetic risk score and cardiovascular events in women. JAMA 303:631-637

3. Ridker PM, Cook NR, Cheng S et al (2003) Alanine for proline substitution in the peroxisome proliferator-activated receptor gamma-2 (PPARG2) gene and the risk of incident myocardial infarction. Arterioscler Thromb Vasc Biol 23:859-863
4. Day N, Oakes S, Luben R et al (1999) EPIC-Norfolk: study design and characteristics of the cohort. European Prospective Investigation of Cancer. Br J Cancer 80(Suppl 1):95-103

5. Patel PS, Sharp SJ, Luben RN et al (2009) Association between type of dietary fish and seafood intake and the risk of incident type 2 diabetes: the European Prospective Investigation of Cancer (EPIC)-Norfolk Cohort Study. Diabetes Care 32:1857-1863

6. Boekholdt SM, Peters RJ, Day NE et al (2004) Macrophage migration inhibitory factor and the risk of myocardial infarction or death due to coronary artery disease in adults without prior myocardial infarction or stroke: the EPIC-Norfolk Prospective Population Study. Am J Med 117:390-397

7. Broadbent HM, Peden JF, Lorkowski S et al (2008) Susceptibility to coronary artery disease and diabetes is encoded by distinct, tightly linked SNPs in the ANRIL locus on chromosome 9p. Hum Mol Genet 17:806-814

8. Harismendy O, Notani D, Song X et al (2011) 9p21 DNA variants associated with coronary artery disease impair interferon-gamma signalling response. Nature 470:264-268

9. Doria A, Wojcik J, Xu R et al (2008) Interaction between poor glycemic control and 9p21 locus on risk of coronary artery disease in type 2 diabetes. JAMA 300:2389-2397

10. Samani NJ, Erdmann J, Hall AS et al (2007) Genomewide association analysis of coronary artery disease. N Engl J Med $357: 443-453$ 